\title{
BAM15, a Mitochondrial Uncoupling Agent, Attenuates Inflammation in the LPS Injection Mouse Model: An Adjunctive Anti-Inflammation on Macrophages and Hepatocytes
}

\author{
Cong Phi Dang ${ }^{\mathrm{a}, \mathrm{b}}$ Jiraphorn Issara-Amphorn ${ }^{\mathrm{b}}$ Awirut Charoensappakit ${ }^{\mathrm{b}}$ \\ Kanyarat Udompornpitak ${ }^{\mathrm{b}}$ Thansita Bhunyakarnjanarat ${ }^{\mathrm{b}}$ Wilasinee Saisorn $^{\mathrm{b}}$ \\ Kritsanawan Sae-Khow ${ }^{\text {b }}$ Asada Leelahavanichkul b,c \\ a Medical Microbiology, Interdisciplinary and International Program, Graduate School, Chulalongkorn University, \\ Bangkok, Thailand; 'Department of Microbiology, Faculty of Medicine, Chulalongkorn University, Bangkok, \\ Thailand; ' Department of Microbiology, Translational Research in Inflammation and Immunology Research Unit \\ (TRIRU), Chulalongkorn University, Bangkok, Thailand
}

\section{Keywords}

BAM15 · Mitochondrial uncoupling · Macrophage $\cdot$

Inflammation · Metabolism

\begin{abstract}
Controlof immune responses through the immunometabolism interference is interesting for sepsis treatment. Then, expression of immunometabolism-associated genes and BAM15, a mitochondrial uncoupling agent, was explored in a proinflammatory model using lipopolysaccharide (LPS) injection. Accordingly, the decreased expression of mitochondrial uncoupling proteins was demonstrated by transcriptomic analysis on metabolism-associated genes in macrophages (RAW246.7) and by polymerase chain reaction in LPS-stimulated RAW246.7 and hepatocytes (Hepa 1-6). Pretreatment with BAM 15 at $24 \mathrm{~h}$ prior to LPS in macrophages attenuated supernatant inflammatory cytokines (IL-6, TNF-a, and IL-10), downregulated genes of proinflammatory M1 polarization (iNOS and IL-1 $\beta$ ), upregulated anti-inflammatory M2 polarization (Arg1 and FIZZ), and decreased cell energy status (extracellular flux analysis and ATP production). Likewise, BAM15 decreased expression of proinflammatory genes (IL-6, TNF- $a$, IL-10, and iNOS) and reduced cell energy
\end{abstract}

karger@karger.com www.karger.com/jin

Karger"

BOPEN ACCESS
(C) 2021 The Author(s)

Published by S. Karger AG, Basel

This is an Open Access article licensed under the Creative Commons Attribution-NonCommercial-4.0 International License (CC BY-NC) (http://www.karger.com/Services/OpenAccessLicense), applicable to the online version of the article only. Usage and distribution for commercial purposes requires written permission. in hepatocytes. In LPS-administered mice, BAM15 attenuated serum cytokines, organ injury (liver enzymes and serum creatinine), and tissue cytokines (livers and kidneys), in part, through the enhanced phosphorylated aAMPK, a sensor of ATP depletion with anti-inflammatory property, in the liver, and reduced inflammatory monocytes/macrophages (Ly6C +ve, $(D 11 b+v e)$ in the liver as detected by Western blot and flow cytometry, respectively. In conclusion, a proof of concept for inflammation attenuation of BAM 15 through metabolic interference-induced anti-inflammation on macrophages and hepatocytes was demonstrated as a new strategy of anti-inflammation in sepsis. @ 2021 The Author(s).

Published by S. Karger AG, Basel

\section{Introduction}

Sepsis, a syndrome of organ dysfunction due to the imbalance of host responses against systemic infection [1], is an important world-wide healthcare problem that is a major cause of death in patients with clinical illness [1]. The shift in the balance toward proinflammatory immune responses, referred to as "hypercytokine syndrome" or "systemic inflammatory response syndrome," is an im- 
portant cause of death in sepsis leading to the utilization of anti-inflammatory adjunctive treatment [2-4]. The robust responses of innate immune cells, including macrophages, mainly account for the sepsis pathophysiology [5].

The plasticity of macrophages, the important immune cells in response to infection [6], classified into a classical activated proinflammatory M1 macrophage polarization and an alternative anti-inflammatory M2 macrophage polarization is well known [7]. An imbalance between M1 and M2 macrophage polarization is responsible for several abnormalities including the prominent M1 macrophage polarization in sepsis-induced hyperinflammatory responses [5]. As such, the blockage of M1 polarization attenuates the exaggerated inflammation in systemic infection $[8,9]$. Interestingly, the metabolic activities of immune cells, at least in part, depend on the status of cell energy (adenosine triphosphate; ATP) that is derived from glycolysis and oxidative phosphorylation (OXPHOS) of mitochondria [10, 11]. Accordingly, glycolysis, but not OXPHOS, is important for the metabolism of M1 macrophages in hyperinflammatory responses, possibly due to the reduced oxygen supply for the OXPHOS cycle [12]. The blockade of glycolysis induces anti-inflammatory status in sepsis $[8,13,14]$. Despite the less importance of OXPHOS during proinflammatory sepsis as previously mentioned [12], OXPHOS is important for inflammation in other models [15] as the decreased OXPHOS reduces inflammation in cord-blood macrophages [16], and blockade of ATP synthesis attenuates obesity-induced proinflammation [17].

Normally, OXPHOS is an effective process of ATP production in mitochondria using the electrochemical proton gradient across the mitochondrial inner membrane through ATP synthase [12]. However, some protons do not enter the ATP synthesis process but leak back into the mitochondrial matrix, referred to as "mitochondrial uncoupling," which leads to the decrease in proton gradients and ATP production [18]. Then, mitochondrial uncoupling proteins (UCPs), a group of proteins with a capacity to transfer protons, enhance ATP synthesis and alter several cell metabolic profiles [19]. Nevertheless, the proton carried by mitochondrial uncoupling agents enhances ATP synthesis only in a very short period of time after the administration but induces an impairment of mitochondrial membrane potential (MMP) that dissipates the proton gradient and reduces ATP synthesis [2022]. Therefore, mitochondrial uncoupling agents are used for ATP production blockage that attenuate macrophage proinflammation [23-25]. Accordingly, the en- hanced mitochondrial UCP2 in transgenic mice attenuates inflammatory cytokines in an ischemic brain model [26], and UCP2 depletion amplifies inflammation

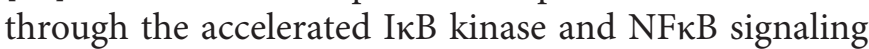
[27]. In parallel, BAM15, a synthetic mitochondrial uncoupling agent, has been used as an interesting candidate of anti-inflammation in several models including ischemic kidney injury [28] and obesity-induced inflammation [29], but never been tested in sepsis. Due to the higher BAM15 distribution in the liver, BAM15 attenuated fatty acid-induced hepatocyte injury (steatohepatitis) in an obesity model [30]. Because the liver is also an important organ that is responsible for inflammatory responses in systemic infection [31], BAM15 might effectively attenuate sepsis-induced liver injury. Different from carbonyl cyanide-4-phenylhydrazone (FCCP), a classical mitochondrial uncoupling agent, BAM15 is more specific to the mitochondrial membrane without an adverse effect on cell membrane depolarization [28]. Here, BAM15 was tested in vitro (macrophages and hepatocytes) and in the lipopolysaccharide (LPS)-induced inflammation mouse model.

\section{Materials and Methods}

\section{Animal and Animal Model}

The animal procedure, in accordance with the protocol of the National Institutes of Health (NIH; USA), was approved by the Institutional Animal Care and Use Committee of the Faculty of Medicine, Chulalongkorn University, Bangkok, Thailand. Male 6-week-old mice, purchased from Nomura Siam International (Pathumwan, Bangkok, Thailand), were housed in the animal facility for 1 week before performing the experiments. Systemic inflammation was induced by endotoxin (LPS) from Escherichia coli 026:B6 (Sigma-Aldrich, St. Louis, MO, USA) as previously described $[8,32,33]$ with BAM15 pretreatment in a dose that was modified from a previous publication [30]. In brief, BAM15 (Sigma-Aldrich) at $1 \mathrm{mg} / \mathrm{kg}$ in $10 \%$ of dimethylsulfoxide (DMSO) (Sigma-Aldrich) or 10\% DMSO alone was intraperitoneally administered at $3 \mathrm{~h}$ prior to the intraperitoneal injection of LPS (4 $\mathrm{mg} / \mathrm{kg}$ ) in phosphate buffer solution (PBS) or PBS alone. Because of the maximal injury at $2 \mathrm{~h}$ after LPS injection in the mouse model [8], all mice were sacrificed with cardiac puncture under isoflurane anesthesia with sample collections (blood and organs) at $2 \mathrm{~h}$ after LPS. Organs (snap frozen on liquid nitrogen) and serum were kept in $-70^{\circ} \mathrm{C}$ until use.

\section{Analysis of Mouse Samples and Western Blotting}

Systemic inflammation was determined by serum cytokines (IL-6, IL-10, and TNF- $\alpha$ ) using ELISA assay (ReproTech, Oldwick, NJ, USA). Liver injury was evaluated by aspartate transaminase (AST) and alanine transaminase (ALT) by using EnzyChrom AST (EASTR-100) and EnzyChrom ALT (EALT-100) (BioAssay, Hayward, CA, USA), respectively. Kidney injury was measured by cre- 
Table 1. List of the primers for macrophage polarization and glycolysis pathway

\begin{tabular}{|c|c|c|}
\hline \multicolumn{3}{|l|}{ Primers } \\
\hline \multirow[t]{2}{*}{$\beta$-Actin } & Forward & 5'-CGGTTCCGATGCCCTGAGGCTCTT-3' \\
\hline & Reverse & 5'-CGTCACACTTCATGATGGAATTGA-3' \\
\hline \multirow[t]{2}{*}{ Uncoupling protein 2} & Forward & 5'-GCCACTTCACTTCTGCCTTC-3' \\
\hline & Reverse & 5'-GAAGGCATGAACCCCTTGTA-3' \\
\hline \multirow[t]{2}{*}{ Uncoupling protein 3} & Forward & 5'-ACCTGGACTGCATGGTAAGG-3' \\
\hline & Reverse & 5'-CTCGTTCTTGCCCTAAGGTG-3' \\
\hline \multirow[t]{2}{*}{ Inducible nitric oxide synthase } & Forward & 5'-CCCTTCCGAAGTTTCTGGCAGCAGC-3' \\
\hline & Reverse & 5'-GGCTGTCAGAGCCTCGTGGCTTTG-3' \\
\hline \multirow[t]{2}{*}{ Arginase 1} & Forward & 5'-CAGAAGAATG GAAGAGTCAG-3' \\
\hline & Reverse & 5'-CAGATATGCA GGGA GTCACC-3' \\
\hline \multirow[t]{2}{*}{ Found in inflammatory zone } & Forward & 5'-GCCAGGTCCTGGAACCTTTC-3' \\
\hline & Reverse & 5'-GGAGCAGGGAGATGCAGATGAG-3' \\
\hline \multirow[t]{2}{*}{ Tumor necrosis factor $a$} & Forward & 5'-CСТCACACTCAGATCATCTTCTC-3' \\
\hline & Reverse & 5'-AGATCCATGCCG TTGGCCAG-3' \\
\hline \multirow[t]{2}{*}{ Interleukin-1 $\beta$} & Forward & 5'-GAAATGCCACCTTTTGACAGTG-3' \\
\hline & Reverse & 5'-TGGATGCTCTCATCAGGACAG-3' \\
\hline \multirow[t]{2}{*}{ Interleukin-6 } & Forward & 5'-CTTCCATCCAGTTGCCTTCT-3' \\
\hline & Reverse & 5'-CCTTCTGTGACTCCAGCTTATC-3' \\
\hline \multirow[t]{2}{*}{ Interleukin-10 } & Forward & 5'-GCTCTTACTGACTGGCATGAG-3' \\
\hline & Reverse & 5'-CGCAGCTCTAGGAGCATGTG-3' \\
\hline
\end{tabular}

atinine (Cr) with QuantiChrom Creatinine-Assay (DICT-500) (BioAssay). Additionally, liver and kidney injury was also evaluated by tissue cytokines following a previous protocol [34]. In brief, tissue samples were weighed and sonicated thoroughly, and the supernatant from homogenous tissue preparation was collected for cytokine measurement by ELISA assay (Biolegend, San Diego, CA, USA) as the serum samples.

Furthermore, protein abundance of phosphorylated AMP-activated protein kinase in alpha isoform ( $\mathrm{p}$ - $\mathrm{AAMPK})$, a molecule for ATP restoration [35], in the liver was examined by Western blot analysis according to a previous protocol [35]. In brief, the liver was homogenized and incubated in lysis buffer (radioimmunoprecipitation assay buffer) complemented with protease and phosphatase inhibitor (Thermo-Scientific, Rockford, IL, USA). Quantification of protein concentration was performed via Bradford assay before protein separation using polyacrylamide gel with $10 \%$ sodium dodecyl sulfate. Thereafter, protein from the gel was transferred to the PVDF membrane, followed by incubation of primary antibodies (Cell Signaling Technology Corporate, Danvers, MA) for (i) p-aAMPK (Thr172) (40H9) (p-aAMPK), (ii) total aAMPK, and (iii) $\beta$-actin (a housekeeping protein) with a second antibody conjugated to horseradish peroxidase and visualized by chemiluminescence (Thermo-Scientific). Notably, p-aAMPK (Thr172) detects endogenous aAMPK only when phosphorylated at threonine 172 , but not at the phosphorylation at threonine 183 that targeted both $\alpha 1$ and $\alpha 2$ isoforms of the catalytic subunit but not the $\beta$ and $\gamma$ regulatory subunits in accordance with the manufacturer's information.

BAM15 Attenuates Inflammation in Macrophages and Hepatocytes
Flow Cytometry Analysis of Immune Cells in the Liver

Flow cytometry analysis of liver macrophages was modified from a previous publication [36-38]. In brief, at $2 \mathrm{~h}$ following LPS injection, livers were minced, homogenized in Roswell Park Memorial Institute media, passed through cell strainers, and incubated with red blood cell lysis $(\mathrm{NH} 4 \mathrm{Cl})$ buffer before washing with PBS. Suspended hepatocytes were preincubated with bovine serum albumin with $2 \%$ heat-inactivated fetal bovine serum for 10 min at room temperature before incubation with antibodies against monocytes/macrophages including FITC-conjugated antiLy6G (BD Biosciences, Franklin Lakes, NJ, USA), phycoerythrinconjugated anti-F4/80 (Biolegend), and allophycocyanin-conjugated anti-CD11b (Biolegend) (at a dilution of 1:100). Then, cells were fixed with $4 \%$ paraformaldehyde (BD Biosciences) and absolute cell counts determined using an absolute count bead (Beckman Coulter, Brea, CA, USA). Flow cytometry data were analyzed by using a BD LSRII cytometer (BD Biosciences) and FlowJo software.

The in vitro Experiments, RNA Sequencing Analysis, and Quantitative Polymerase Chain Reaction

The cell lines of murine macrophages (RAW264.7) (TIB-71 ${ }^{\mathrm{TM}}$; ATCC, Manassas, VA, USA) and murine hepatocytes (Hepa 1-6) (CRL-1830 ${ }^{\mathrm{TM}}$, ATCC) were incubated in Dulbecco's Modified Eagle Medium (DMEM) with penicillin-streptomycin and heat-inactivated fetal bovine serum (Thermo Fisher Scientific, Waltham, MA, USA) at $37^{\circ} \mathrm{C}$ with $5 \%$ carbon dioxide. Cells were seeded overnight before the pretreatment with BAM15 (different concentra- 
tions and time points) or DMSO control before the activation in macrophages or hepatocytes by LPS $(100 \mathrm{ng} / \mathrm{mL})$ for $6 \mathrm{~h}$ or LPS (1 $\mu \mathrm{g} / \mathrm{mL}$ ) for $1-6 \mathrm{~h}$, respectively, prior to cell harvesting due to the different LPS responses between these cells $[39,40]$. For preparation of heat-killed microbes, E. coli (ATCC 25922), Staphylococcus aureus (ATCC 25923), and Candida albicans (ATCC 14053) (the American Type Culture Collection, Manassas, VA, USA) were heat inactivated at $65^{\circ} \mathrm{C}$ for $30 \mathrm{~min}$ and thoroughly sonicated as in a previous publication [41]. Then, RAW264.7 cells were stimulated with each of these heat-killed microbes with a ratio between RAW264.7 cells and each organism (cells) at 1:10 for $6 \mathrm{~h}$ before the sample collection. Then, cytokines in the supernatant were measured by ELISA assay (Biolegend), and cells were used for RNA extraction and extracellular flux analysis.

In macrophages, RNA sequencing analysis was performed to explore the downstream gene expression after LPS stimulation by the BGI Company. The differential gene expression using R package and the biological process and pathway analysis were performed by GO analysis and gene ontology pathway analysis, respectively [42]. In addition, several interesting molecules in both cell types were determined by qPCR as previously described [33]. In brief, RNA extraction from cells was performed using Trizol (Thermo Fisher Scientific). Amount of RNA was determined by using Nano drop ND-1000 (Thermo Fisher Scientific) before converting to cDNA by using the reverse transcription system. Gene expression was determined by qPCR using the cDNA template, target primers, and SYBR Green master mix (Applied Biosystem, Foster City, CA, USA), and $\beta$-actin was used as a housekeeping gene in the $\Delta \Delta \mathrm{CT}$ method. The primers of target genes are listed in Table 1.

\section{MMP Assessment, Extracellular Flux Analysis, Cellular}

Lactate, and Total Cellular ATP

MMP was investigated using MitoTracker Red CMXRos (Thermo Fisher Scientific) as a red fluorescent color on mitochondria with active membrane potential in accordance with the manufacturer's protocol. In brief, $200 \mathrm{nM}$ of MitoTracker Red was incubated with the cell culture at $37^{\circ} \mathrm{C}$ for $30 \mathrm{~min}$ before fixation with methanol at $20^{\circ} \mathrm{C}$ for $15 \mathrm{~min}$. Cells were also stained with $4^{\prime}, 6$-diamidino-2-phenylindole DAPI (Sigma Aldrich), the blue fluorescent color DNA staining. The fluorescent images were photographed by using the confocal microscope ZEISS LSM 800 (Carl Zeiss, Germany) with the intensity analysis using the Varioskan Flash microplate reader (Thermo Fisher Scientific). Oxygen consumption rate (OCR) and extracellular acidification rate (ECAR), a representative of mitochondrial function (respiration) and glycolysis, respectively, were analyzed by using Seahorse XFp Analyzers (Agilent, Santa Clara, CA, USA) as described in a previous publication [43]. In brief, cells were stimulated, as described above, in a Seahorse cell culture plate before replacing by Seahorse media (DMEM complemented with glucose, pyruvate, and L-glutamine) (Agilent, 103575-100) in $\mathrm{pH} 7.4$ at $37^{\circ} \mathrm{C}$ for $1 \mathrm{~h}$ prior to the challenge with different metabolic interference compounds including oligomycin $1.5 \mu \mathrm{M}$, carbonyl cyanide-4-(trifluoromethoxy)-phenylhydrazone (FCCP) $1 \mu \mathrm{M}$, and rotenone/antimycin A $0.5 \mu \mathrm{M}$ according to the protocol. The ECAR graph was calculated from OCR data, and all data were analyzed by using Seahorse Wave 2.6 software based on the following equations: basal respiration = OCR before oligomycin - OCR after rotenone/antimycin A, maximal respiration $=$ OCR between FCCP and rotenone/antimycin
$\mathrm{A}$ - OCR after rotenone/antimycin $\mathrm{A}$, and respiratory reserve $=$ OCR between FCCP and rotenone/antimycin A - OCR before oligomycin. In addition, cellular lactate by colorimetric assay (BioVision, Milpitas, CA 95035, USA) and cellular ATP analysis by luminescent ATP detection assay (Abcam, Cambridge, UK) were performed according to the manufacturer's protocol.

\section{Statistical Analysis}

Graph establishment and data analysis was performed using GraphPad Prism 5.0 (GraphPad Software, Inc., San Diego, CA, USA) and demonstrated in mean \pm standard error (SEM). Student's $t$ test or one-way analysis of variance upon Tukey's analysis was used to determine statistical significance between 2 or more groups, respectively. Statistical significance was marked with the $p$ value $<0.05$

\section{Results}

Pretreatment with BAM15 reduced inflammatory responses in LPS-stimulated macrophages (RAW264.7) and hepatocytes (Hep1-6), and BAM15 attenuated the severity of LPS-injected mice.

\section{The Reduced Mitochondrial Uncoupling-Related \\ Genes in LPS-Activated Macrophages: An \\ Association between Cell Energy and Macrophage Proinflammatory Responses}

To investigate a possible association between cell energy and inflammatory responses in macrophages, the RNA sequencing analysis of metabolism-associated genes in macrophages after stimulation by LPS or PBS control was performed. Accordingly, a reduction in most genes with an increase in a few genes was demonstrated in LPSstimulated macrophages compared with the control (Fig. 1a). The UCPs (UCP1, UCP2, and UCP3), ATP synthesis-associated molecules, were among the LPS-downregulated genes, indicating a reduced mitochondrial function after LPS stimulation (Fig. 1a). Although most of the genes in the respiratory electron transport and tricarboxylic acid cycle were downregulated, some genes including LDHA, SLC16A3, and PDK3, pyruvate metabolism-associated genes [44], were upregulated suggesting a compensatory increased glucose consumption during LPS-induced mitochondrial dysfunction to restore cell energy status.

Due to the hepatic impacts in severe inflammatory responses $[31,45,46]$ and the altered metabolic profiles in activated macrophages [43], LPS was stimulated in both macrophages and hepatocytes to further explore UCP gene expression. Indeed, a downregulation of both UCP2 and UCP3 was also demonstrated in both macrophages 


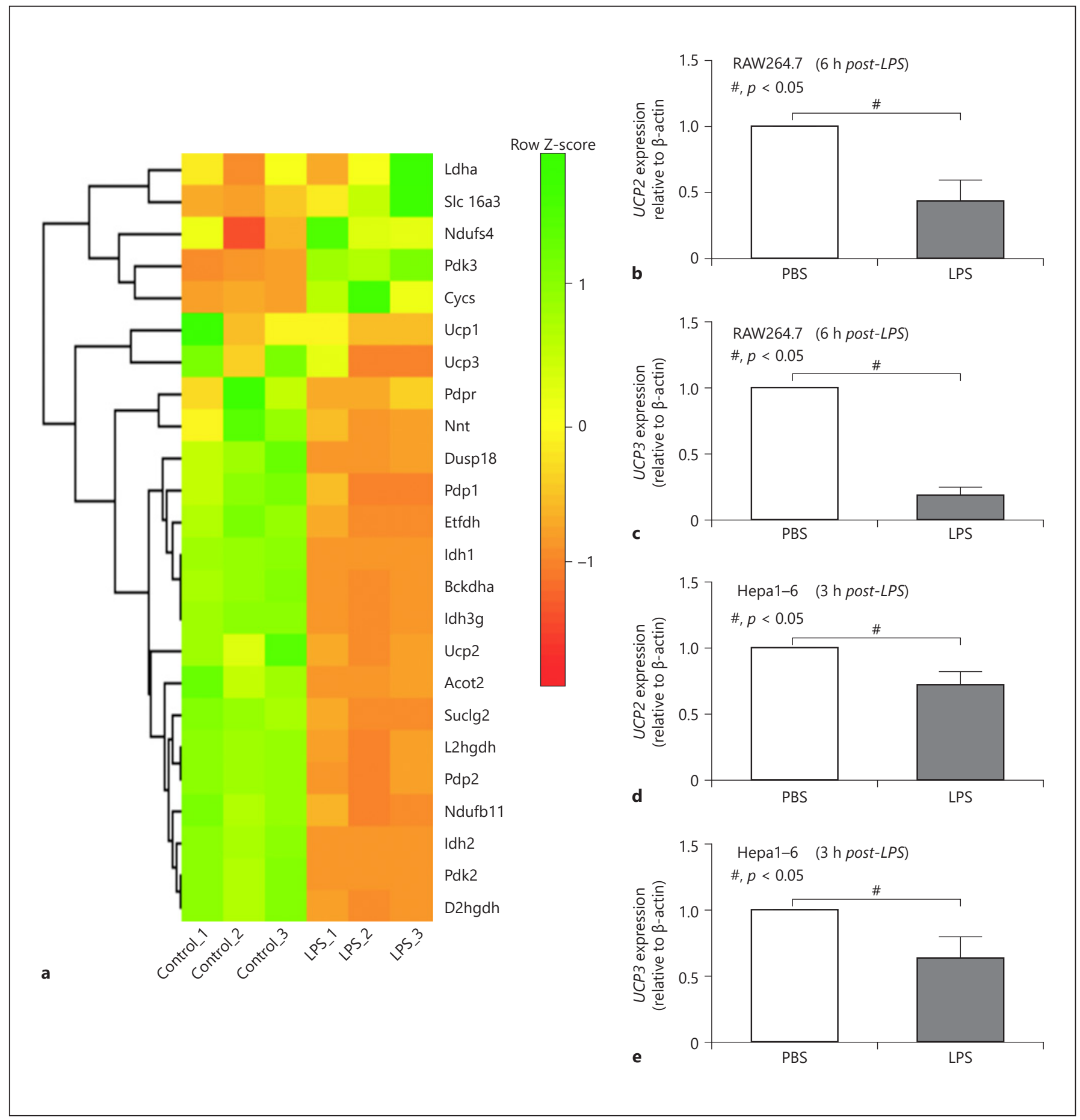

Fig. 1. The transcriptome analysis in macrophages (RAW264.7) and UCP gene expression in LPS-stimulated RAW264.7 and hepatocytes (Hepa 1-6). The transcriptome analysis in macrophages (RAW264.7) presented by hierarchical clustering heat-map profiling groups focusing on OXPHOS and mitochondrial respirationassociated genes (a) and the gene expression of mitochondrial
UCPs (UCP2 and UCP3) in macrophages and hepatocytes (Hepa 1-6) (b-e) as determined by qPCR relative to expression of the $\beta$-actin housekeeping gene are demonstrated (independent triplicate experiments were performed). LPS, lipopolysaccharide; UCPs, uncoupling proteins; OXPHOS, oxidative phosphorylation; qPCR, quantitative polymerase chain reaction. 


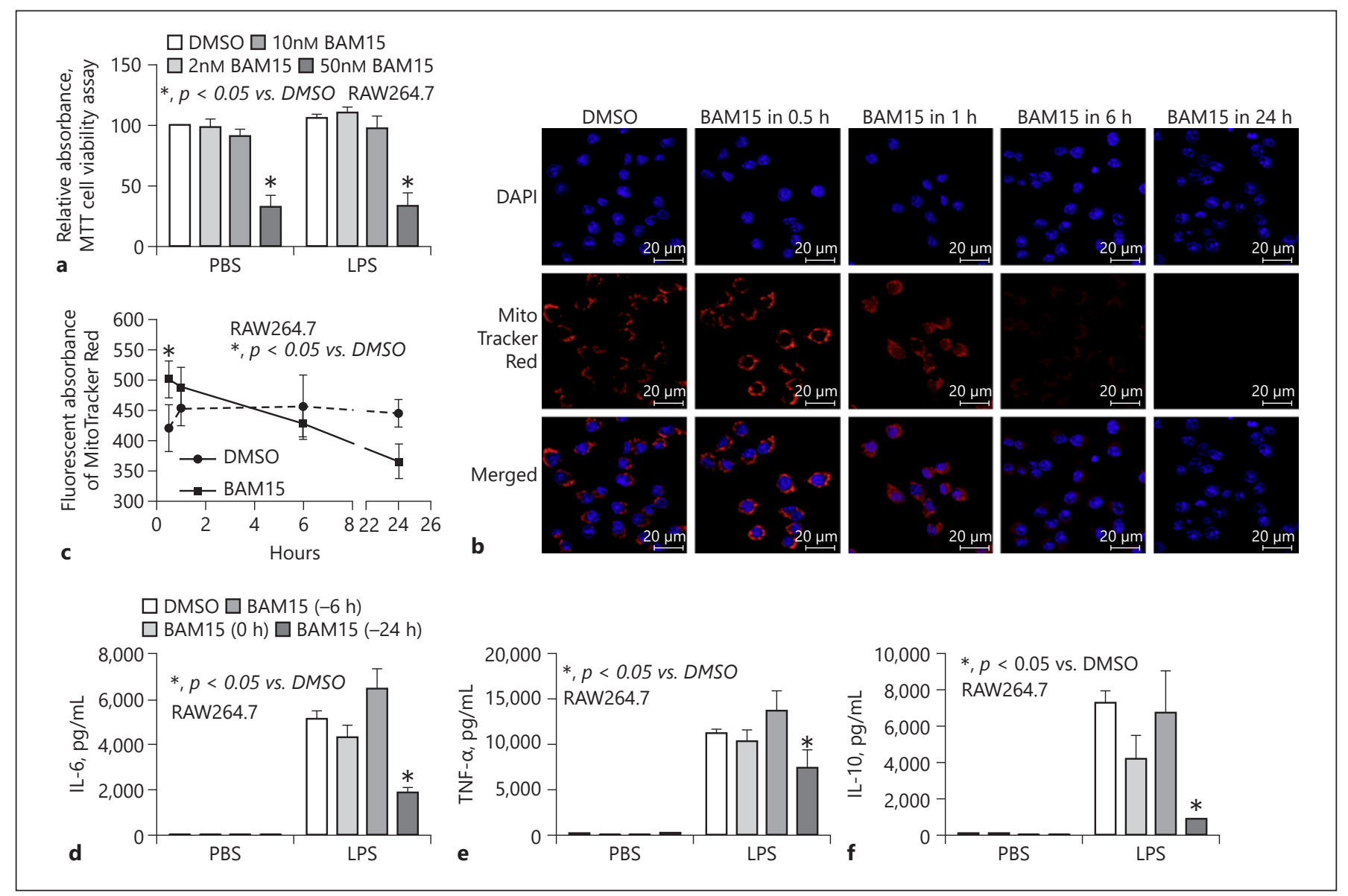

Fig. 2. The in vitro optimization of BAM15 on macrophages (RAW264.7). BAM15 or DMSO in the different concentrations was incubated with macrophages for $24 \mathrm{~h}$ before cell viability was tested with tetrazolium 3-(4, 5-dimethylthiazol-2-yl)-2, 5-diphenyltetrazolium assay (a). MMP measurement in different times of BAM15 incubation by MitoTracker Red CMXRos as demonstrat-

and hepatocytes after LPS stimulation (Fig. 1a-e). However, UCP1 was nondetectable in both cells (data not shown). Due to the decreased expression of UCPs (UCP2 and UCP3) during LPS stimulation, a supplement with mitochondrial uncoupling agents might be beneficial in the hyperinflammatory status.

\section{BAM15 Reduced Inflammatory Responses (Cytokines} and Proinflammatory M1 Macrophage Polarization) through the Interference in Macrophage Metabolic Profiles

According to the cell viability assay using tetrazolium 3-(4,5-dimethylthiazol-2-yl)-2,5-diphenyltetrazolium, BAM15 at the concentrations $<50 \mathrm{nM}$ was nontoxic to macrophages (Fig. 2a). Then, BAM15 at $10 \mathrm{nM}$ was fur- ed by the representative fluorescent pictures with the fluorescent absorbance score $(\mathbf{b}, \mathbf{c})$ and the supernatant cytokines from macrophages with the pretreatment of BAM15 $(10 \mathrm{nM})$ at the different time points before LPS administration (d-f) are demonstrated (independent triplicate experiments were performed). MMP, mitochondrial membrane potential; LPS, lipopolysaccharide.

ther used in all of the in vitro experiments. The different incubation times of BAM15 were tested in RAW264.7 cells to investigate the kinetic effect of BAM15 on MMP as determined by the staining with MitoTracker Red. As expected, BAM15 enhanced MMP at the early time point ( 0.5 and $1 \mathrm{~h}$ after BAM15) and then downregulated at 24 $\mathrm{h}$ after BAM15 with the reduction of supernatant cytokines (Fig. 2b-f). At $24 \mathrm{~h}$ after BAM15, there was reduction in supernatant cytokines (IL-6, TNF- $\alpha$, and IL-10) (Fig. $3 \mathrm{a}-\mathrm{c}$ ) and proinflammatory markers of M1 polarization (iNOS and IL-1 $\beta$ ) with the enhancement in antiinflammatory M2 polarization at $6 \mathrm{~h}$ after LPS in macrophages (Fig. 3e-h). Moreover, BAM15 also attenuated inflammatory responses after activation by the heatkilled microbes, including E. coli, S. aureus, and C. albi- 


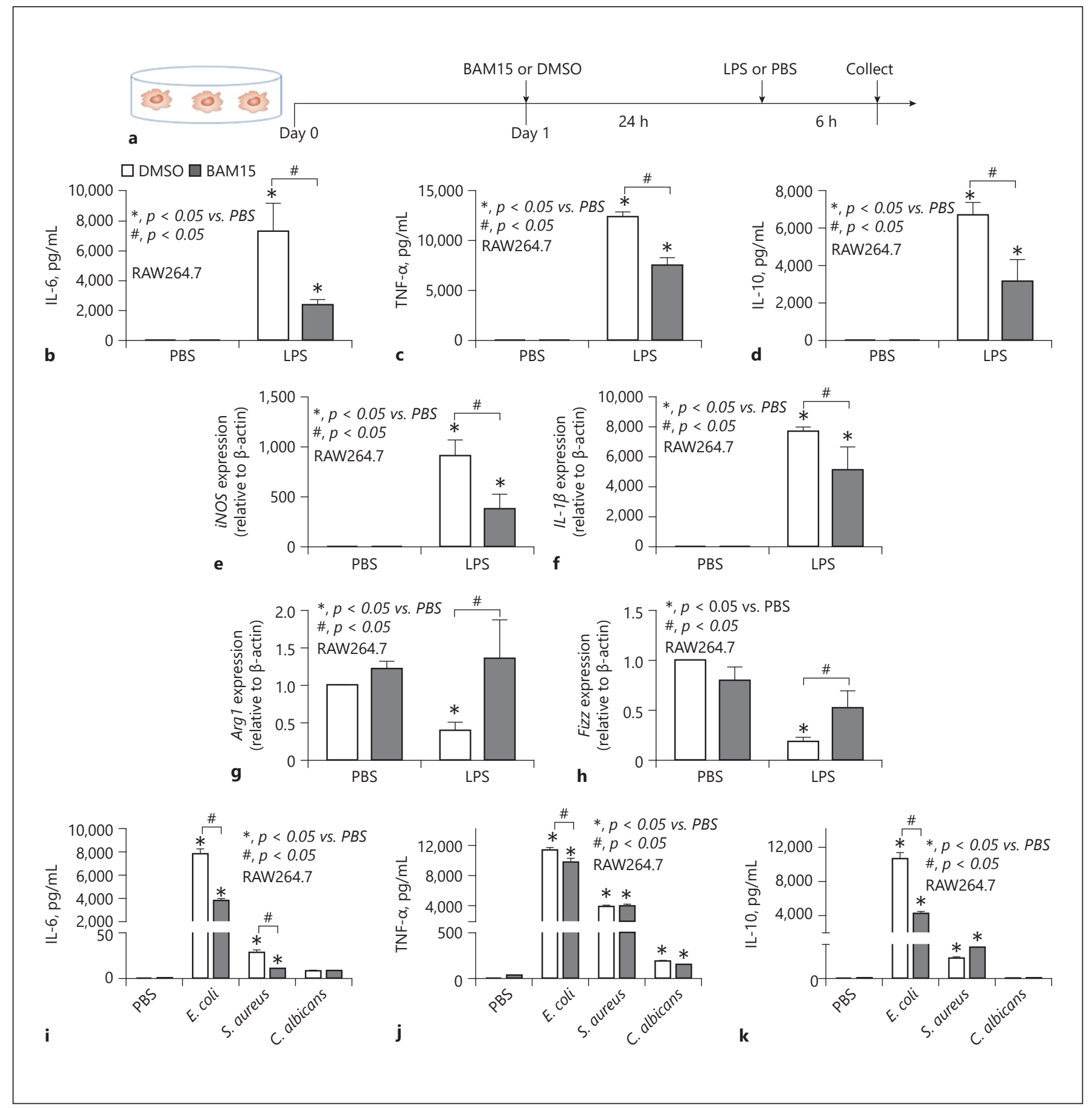

Fig. 3. The anti-inflammatory effect of BAM15 in macrophages (RAW264.7). The responses of macrophages (RAW264.7) with 24-h BAM15 (or DMSO control) pretreatment prior to 6-h LPS stimulation (or PBS) as displayed by the schematic diagram (a) and evaluated by supernatant cytokines (b-d) and gene expression of proinflammatory M1 (iNOS and IL-1 $\beta$ ) or anti-inflammatory M2 (Arg-1 and Fizz) macrophage polarization using qPCR relative to expression of the $\beta$-actin housekeeping gene (e-h) are demonstrated (independent triplicate experiments were performed). In similar experimental designs, the responses of macrophages against the heat-killed microbes including Escherichia coli, Staphylococcus aureus, and Candida albicans (i-k) are also demonstrated (independent triplicate experiments were performed). LPS, lipopolysaccharide; qPCR, quantitative polymerase chain reaction. 
cans despite the difference in proinflammatory activity (Fig. 3i-k).

In parallel, 6-h LPS stimulation decreased mitochondrial functions (OCR) but enhanced glycolysis activity (ECAR) when compared with PBS negative control (Fig. 4a, b), supporting the glycolysis-associated proinflammatory macrophages $[13,47]$. The reduction of mitochondrial activity after LPS stimulation was also indicated by the decrease in several extracellular flux analysis parameters including basal respiration, maximal respiration (a short period of mitochondrial activity enhancement with FCCP), and respiratory reserve (an ability to enhance mitochondrial activity from the baseline level) (Fig. 4c-e). Due to the increased proinflammatory activities with decreased mitochondrial function in LPS-stimulated macrophages, mitochondrial ATP production and total ATP in macrophages were decreased (Fig. 4f, g) along with a compensatory increased glycolysis as indicated by increased lactate (Fig. 4h), an end product of glycolysis [48]. Then, BAM15 reduced cell energy in macrophages with LPS or PBS (Fig. 4a-h), but BAM15 decreased cytokine production and proinflammatory features only in LPS-stimulated cells, but not control cells with PBS (Fig. 3b-k).

\section{BAM15 Interfered with Hepatocyte Metabolic Profiles and Attenuated Septic Severity in LPS Injection \\ Mouse Model, Partly through an Enhanced AMPK in the Liver}

Because of the liver accumulation of BAM15 [30] and the importance of hepatocytes in proinflammatory sepsis [31], effect of BAM15 on hepatocytes was evaluated. Indeed, LPS-induced inflammatory cytokines and iNOS expression in hepatocytes (Fig. 5a-c) supported the LPS inflammatory activation [40]. Then, BAM15 attenuated these parameters, but not anti-inflammatory IL-10 (Fig. $5 \mathrm{a}-\mathrm{c}$ ). In parallel, LPS reduced mitochondrial function (OCR) in hepatocytes (Fig. 6a-e), similar to LPS-stimulated macrophages (Fig. 4a-e), but LPS did not enhanced hepatic glycolysis activity (ECAR) (Fig. 6a-e), different from LPS-stimulated macrophages (Fig. 4a-e), perhaps due to hepatic glycogen storage. Additionally, LPS reduced

Fig. 4. The cell energy analysis of LPS-stimulated macrophages (RAW264.7) with BAM15 pretreatment. The extracellular flux analysis pattern of macrophages (RAW264.7) with 24-h BAM15 (or DMSO control) pretreatment prior to 6-h LPS stimulation (or PBS) as evaluated by OCR of the mitochondrial stress test for mitochondrial pathway analysis (a) and ECAR of the glucose stress test for glycolysis pathway analysis (b) with the parameters of mitochondrial activities including basal respiration, maximal respi- basal respiration, maximal respiration, respiratory reserve, and mitochondrial ATP production (Fig. $6 \mathrm{c}-\mathrm{f}$ ) but did not decrease total hepatocyte ATP and lactate production (Fig. $6 \mathrm{~g}, \mathrm{~h}$ ), different from LPS-stimulated macrophages (Fig. $4 \mathrm{~g}, \mathrm{~h}$ ), possibly due to the prominent compensation by potent hepatic glycolysis $[49,50]$. BAM15 partly reduced hepatocyte mitochondrial function (decreased glycolysis reserve but not basal and maximal respiration) and decreased other metabolic profiles (glycolysis activity, mitochondrial ATP production, total hepatocyte ATP, and lactate production) (Fig. $6 \mathrm{c}-\mathrm{h}$ ). Hence, BAM15 attenuated inflammatory responses both in macrophages and hepatocytes through the cell-energy interference.

In correspondence to the in vitro anti-inflammation, BAM15 attenuated systemic inflammatory cytokines (IL6 and TNF- $\alpha$ ) but not anti-inflammatory (IL-10) along with liver and kidney injury (liver enzymes and serum $\mathrm{Cr}$ ) (Fig. 7a-f). Because BAM15 is accumulated in the liver and excreted through the kidney [30], the anti-inflammatory effect of BAM 15 in these organs might be more prominent. Indeed, the reduced inflammatory cytokines (IL-6 and TNF- $\alpha$ ) and increased anti-inflammatory IL-10 in the liver and kidney were demonstrated (Fig. 7g-l). Additionally, other systemic injury parameters such as AST, ALT, and $\mathrm{Cr}$ also significantly attenuated with BAM15. Because most of the cell populations in the liver are hepatocytes (liver parenchymal cells) with only 15\% of Kupffer cells (immune cells) and other nonparenchymal cells (supporting cells) [51, 52], the lysate of liver tissue is used as a representative of hepatocytic protein expression. As such, LPS increased hepatic AMP-activated protein kinase (AMPK), an anti-inflammatory metabolic sensor of ATP depletion [53,54] (Fig. 8a), possibly counteracted against the LPS proinflammatory effect (Fig. 5f, g). The higher liver AMPK (Fig. 8a) was another mechanism of BAM15 anti-inflammation in systemic infection. Likewise, BAM15 also reduced hepatic accumulation of inflammatory macrophages (CD11b positive, Ly6C positive), but not the total macrophages (F4/80 positive) of LPS-administered mice (Fig. $8 \mathrm{~b}-\mathrm{d}$ ). Hence, BAM15 is an interesting candidate for anti-inflammatory adjunctive treatment in sepsis.

ration, and mitochondrial reserve (c-e) are demonstrated (independent triplicate experiments were performed). Additionally, the mitochondrial ATP production using the extracellular flux analysis (f), total ATP production (chemiluminescent assay) (g), and supernatant lactate (colorimetric assay) (h) is indicated (independent triplicate experiments were performed). LPS, lipopolysaccharide; OCR, oxygen consumption rate; ECAR, extracellular acidification rate.

(For figure see next page.) 

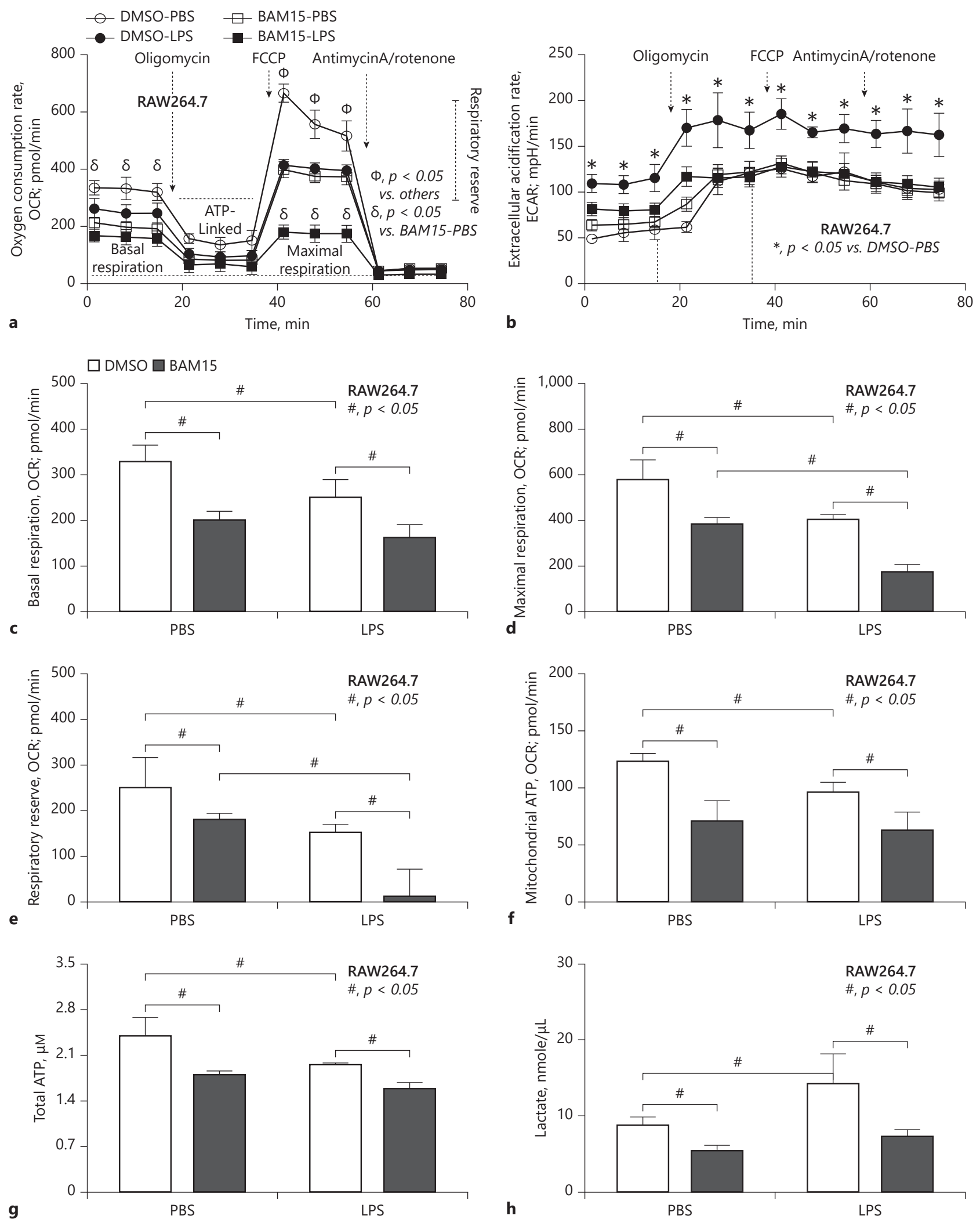


\section{Discussion}

BAM15, a mitochondrial uncoupling agent, attenuated the severity of the LPS injection mouse model through the reduction of mitochondrial activity and cell energy status in macrophages and hepatocytes.
The Downregulation of Mitochondrial UCP after LPS Stimulation: A Possible Natural Balance against LPSInduced Proinflammation

Although mitochondria are an important source of the cellular ATP production through the electrochemical proton-gradient in OXPHOS, the dissipation (leakage) of

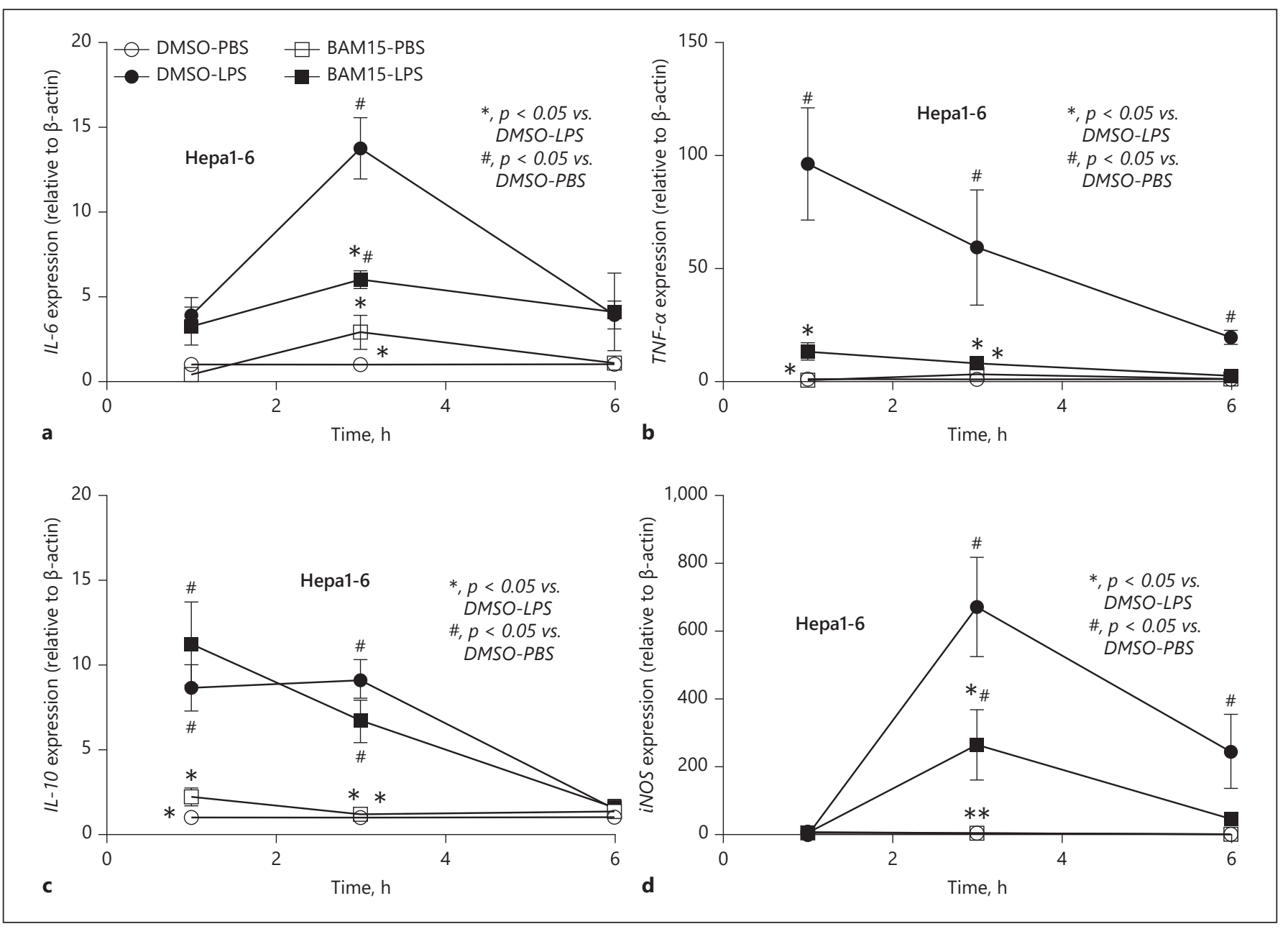

Fig. 5. The anti-inflammatory effect of BAM15 in hepatocytes (Hepa 1-6). The responses of hepatocytes (Hepa 1-6) with 24-h BAM15 (or DMSO control) pretreatment prior to 6-h LPS stimulation (or PBS) as evaluated by gene expression of cytokines (IL-6, TNF- $\alpha$, and IL-10) and iNOS using qPCR relative to expression of the $\beta$-actin housekeeping gene (a-d) are demonstrated (independent triplicate experiments were performed). LPS, lipopolysaccharide; iNOS, inducible nitric oxide synthase; qPCR, quantitative polymerase chain reaction.

Fig. 6. The cell energy analysis of LPS-stimulated hepatocytes (Hepa 1-6) with BAM15 pretreatment. The extracellular flux analysis pattern of hepatocytes (Hepa 1-6) with 24-h BAM15 (or DMSO control) pretreatment prior to 6-h LPS stimulation (or PBS) as evaluated by OCR of the mitochondrial stress test for mitochondrial pathway analysis (a) and ECAR of the glucose stress test for glycolysis pathway analysis (b) with the parameters of mitochondrial activities including basal respiration, maximal respi- ration, and mitochondrial reserve (c-e) are demonstrated (independent triplicate experiments were performed). Additionally, the mitochondrial ATP production using the extracellular flux analysis (f), total ATP production (chemiluminescent assay) (g), and supernatant lactate (colorimetric assay) (h) is indicated (independent triplicate experiments were performed). LPS, lipopolysaccharide; OCR, oxygen consumption rate; ECAR, extracellular acidification rate.
(For figure see next page.) 

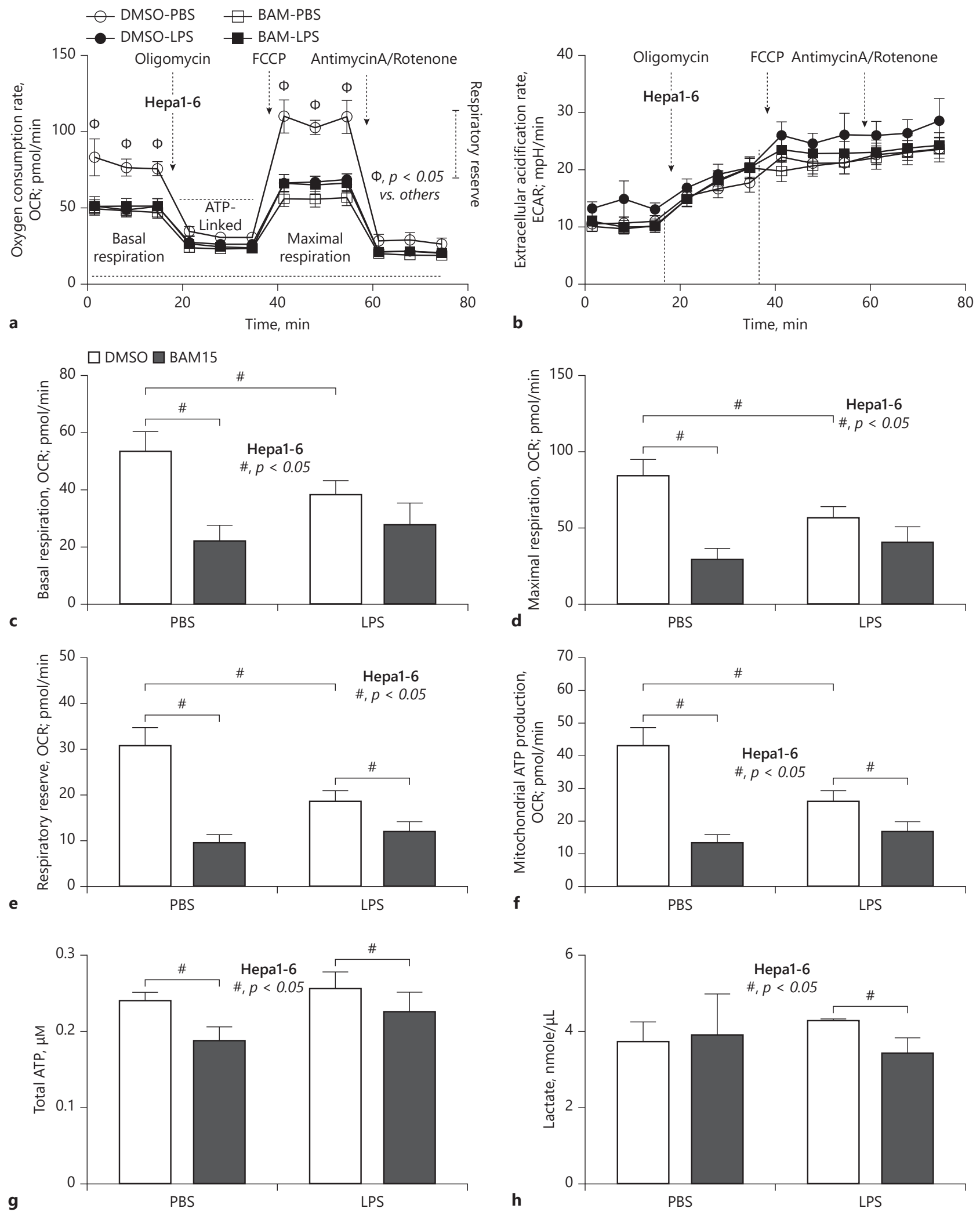

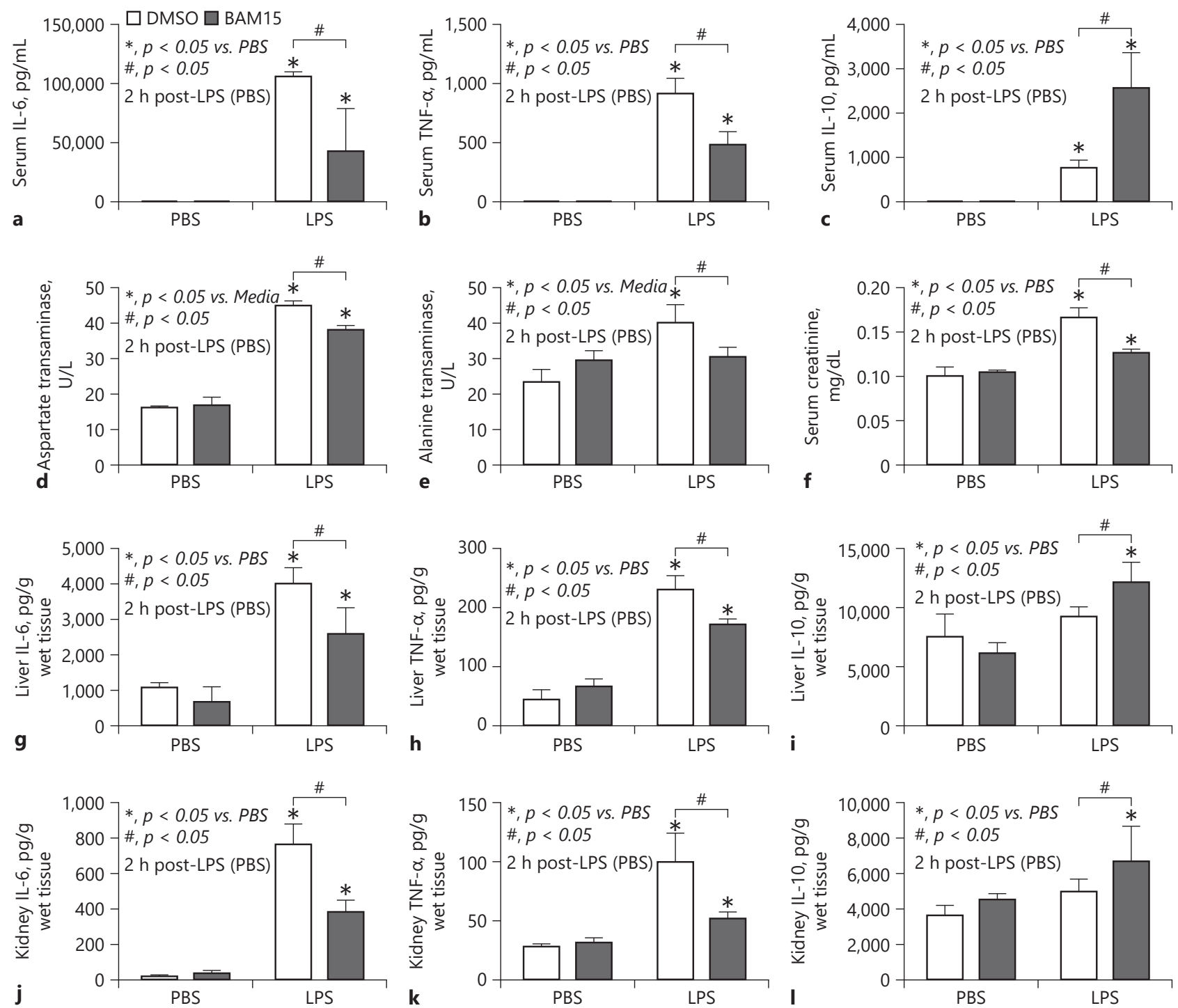

Fig. 7. BAM15 ameliorated the severity of LPS-administered mice. The severity of mice with intraperitoneal injection of BAM15 $(1 \mathrm{mg} / \mathrm{kg})$ or DMSO $(10 \%)$ at $3 \mathrm{~h}$ prior to the intraperitoneal administration of LPS or PBS as evaluated by serum cytokines (a-c), liver enzymes (AST and ALT) (d, e), kidney injury (serum creatinine) (f), and cytokines in livers and kidneys $(\mathbf{g}-\mathbf{I})$ is demonstrated ( $n=6-8 \mathrm{mice} /$ group). LPS, lipopolysaccharide; AST, aspartate transaminase; ALT, alanine transaminase.

Fig. 8. The increased abundance of activated AMPK in the liver and flow cytometry analysis of LPS-administered mice with BAM15 pretreatment. The abundance of hepatic $\mathrm{p}$-aAMPK from mice with intraperitoneal injection of BAM15 $(1 \mathrm{mg} / \mathrm{kg})$ or DMSO $(10 \%)$ at $3 \mathrm{~h}$ prior to intraperitoneal administration of LPS or PBS by Western blot analysis in relative ratio to total aAMPK along with the $\beta$-actin internal control with the representative blotting picture (a) and the flow cytometry analysis from the liver for total and inflam- matory monocytes/macrophages using F4/80 positive (F4/80 +ve) and CD11b positive with Ly6C positive (Ly6C +ve and CD11b $+\mathrm{ve}$ ), respectively, with the representative flow cytometry pattern (b-d) are demonstrated ( $n=4-6 /$ group). The representative flow cytometry pattern of PBS-DMSO was not presented due to the similarity to PBS-BAM15. LPS, lipopolysaccharide; $p$ - $\alpha$ AMPK, phosphorylated AMP-activated protein kinase in alpha isoform.

(For figure see next page.) 

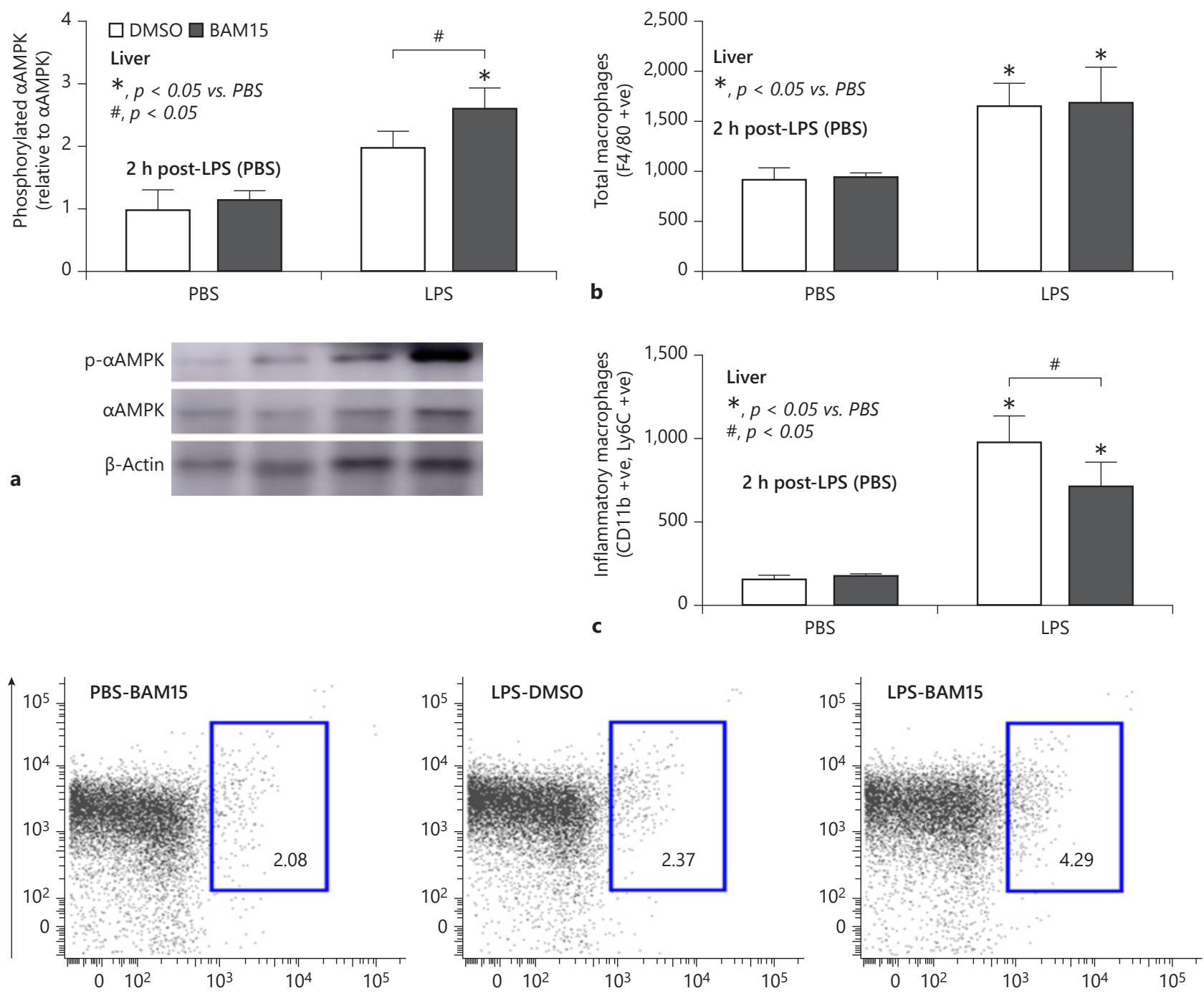

F4/80 PE
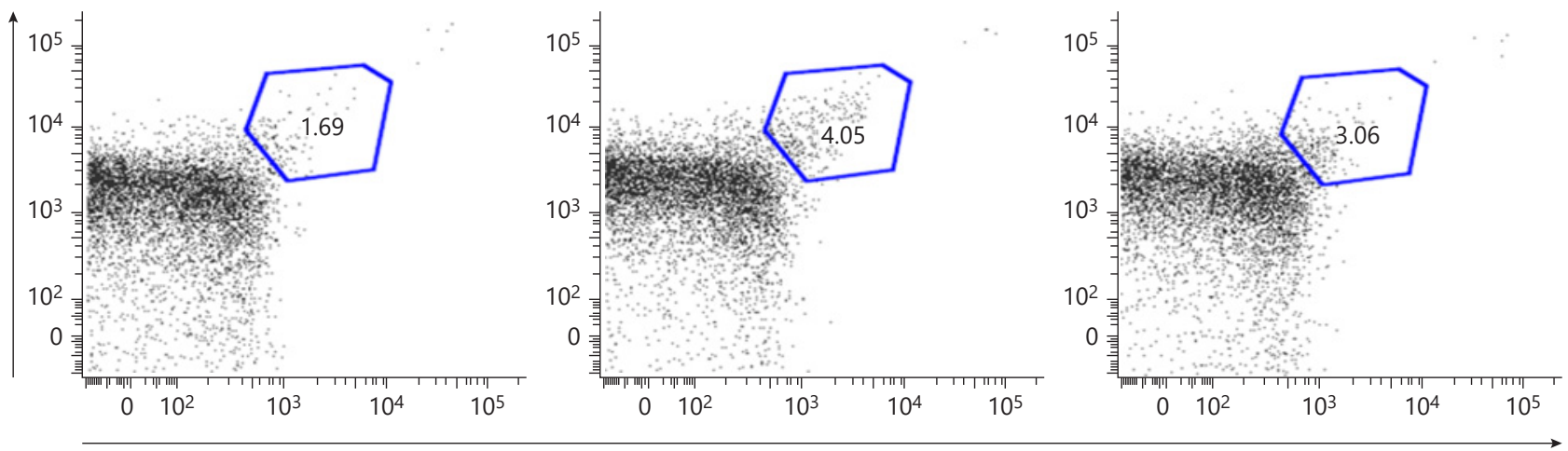

d

Ly6C PerCP Cy5.5 
the proton away from the OXPHOS cycle reduces the effectiveness of ATP synthesis [55]. The leaked protons also increase cell injury from increased reactive oxygen species [25]. Mitochondrial UCPs transfer the proton into the OXPHOS cycle which results in a quick boost-up of ATP synthesis but followed by a rapid ATP reduction [20, $23,56]$ which leads to a reduction of cell responses [18]. As such, UCP2 depletion in the transgenic mice increased IL- $1 \beta$ and IL- 6 [57], and UCP 2 overexpression suppresses IL- 6 and Bcl-2 that attenuates cerebral ischemic injury [26]. Likewise, the decreased cell energy status which leads to the reduction in cytokine production in macrophages is also demonstrated [35].

Here, LPS downregulated UCPs (mitochondrial function), in either macrophages or hepatocytes, but enhanced glycolysis and reduced cell energy status only in macrophages but not in hepatocytes. While LPS could enhance glycolysis in macrophages (glycolysis-dependent macrophage proinflammation) [13, 47], LPS could not further increase hepatic glycolysis possibly because of the lower hepatic cytokine production ability and the more dominant hepatic glycolysis when compared with macrophages $[49,50,58]$. Although the different LPS-activated metabolic responses between macrophages and hepatocytes are out of our scope, these data suggest the prominent role of mitochondrial UCPs in both cells which are a possible target for sepsis immune regulation.

\section{BAM15, a Synthetic Mitochondrial Uncoupling} Agent, Attenuated Inflammation in Macrophages and Hepatocytes by Suppressing Both Mitochondrial

Function and Glycolysis

Hyperinflammatory responses in sepsis which are a main cause of the high mortality and the proinflammatory attenuation, especially against macrophages, are mentioned $[9,59]$. Currently, the metabolism modification which is an interesting strategy to harness macrophage responses as an alteration of the main energy metabolism from mitochondria to glycolysis in proinflammatory M1 macrophages is well known [13, 47]. Not only energy generation and thermogenesis but also an impact of mitochondria toward immune responses is well described [15]. Here, a mitochondrial uncoupling agent (BAM15) attenuated inflammation via the suppression of cell energy status on mitochondria and glycolysis activity, supported by previous publications $[23,60]$. Our model demonstrated that BAM15 increased cell energy status at the early time point $(<1 \mathrm{~h}$ after BAM15) similar to the previous reports $[29,30]$; however, the cell energy was reduced at $24 \mathrm{~h}$ after BAM15 resulting in the anti-inflam- matory characteristics. Hence, this discrepancy might be explained by the difference in dose of BAM15, the activation time of BAM15 treatment, and the cell type used in the studies. These data suggested an importance of the delivery methods for BAM15 administration in the clinical utilization. Perhaps, the theoretically enhanced mitochondrial functions (the energy boost-up) by proton transfer from uncoupling agents might be very transient, and, in contrast, the uncoupling agents might eventually result in mitochondrial energy exhaustion and anti-inflammatory characteristics $[16,17,61,62]$. Indeed, the increased pyruvate influx into the tricarboxylic acid cycle using dichloroacetate results in a more sustainable enhanced OXPHOS, in comparison with the UCPs, and improves bactericidal activity [63] but does not improve clinical outcomes in patients with sepsis [64] possibly due to the increased cytokine production [65]. These data demonstrated the diverse responses between the different strategies on mitochondria. Notably, the organism control by antibiotics during sepsis adjunctive anti-inflammatory treatment is very important as the proinflammatory cytokines are necessary for organismal eradication [66]. Similar to our results with BAM15, the reduced mitochondrial function indirectly decreased glycolysis due to the compensatory increased pyruvate influx into mitochondria which reduced pentose phosphate pathways of glycolysis as demonstrated by the reduced lactate after treatment with niclosamide ethanolamine and oxyclozanide (the mitochondrial uncoupling agents) [67]. Because BAM15 attenuated inflammatory cytokines in both macrophages and hepatocytes and downregulated proinflammatory M1 macrophage polarization, BAM15 was interesting for anti-inflammatory treatment in the hyperinflammatory status.

\section{Administration of BAM15 Attenuated the Severity of} the LPS Injection Mouse Model

Despite the BAM15 anti-inflammatory effect in several models [28-30], BAM15 has never been tested in LPSinduced inflammation. Here, BAM15 attenuates both systemic and local inflammation as indicated by proinflammatory cytokines in serum and in organs (livers and kidneys), respectively, along with liver enzymes and serum Cr. Additionally, the BAM15 anti-inflammatory property was also demonstrated through the nonreduction of IL-10 both in serum and in organs, possibly due to the enhanced anti-inflammatory M2 macrophage polarization from BAM15. Likewise, an administration of UCP2, a natural mitochondrial UCP, also enhances IL-10 and facilitates M2 polarization in microglia and in RAW264.7 [68, 69]. 
Perhaps, the glycolysis reduction did not only downregulate M1 macrophage polarization but also induced M2 polarization during inflammatory activation $[8,13,14]$. The prominent IL-10 in both kidneys and livers in LPS-administered mice was also possibly due to the BAM15 accumulation in both organs [30], which demonstrated a potent anti-inflammatory local effect on both organs, the most common organ failure in severe infection $[31,70,71]$. Since the liver is an important organ associated with sepsis proinflammation (producing acute phase proteins and complements) and thermoregulation [72], the reduced hepatic energy status might activate AMPK, a metabolic sensor for ATP depletion [53, 54]. Indeed, LPS slightly enhanced liver AMPK, and BAM15 further increased liver AMPK which might amplify anti-inflammatory responses [73, 74]. Indeed, AMPK, an activated molecule during the depleted cell energy status, induces several processes to maintain the energy homeostasis and prevents cell damages in several models (sepsis, hepatic ischemic reperfusion, and acute kidney disease) [75-77] through multiple mechanisms, including autophagy induction with apoptosis downregulation, enhancement of the alternative sources for cell energy (lipolysis and fatty acid oxidation), and reduction in oxidative stress and inflammatory signaling [78-82]. Overall, AMPK anti-inflammatory effect is a cell adaptation to reduce the energy utilization (decreased cytokine production) due to an energy insufficient condition [35]. Notably, we did not observe LPSinduced hepatic lipid accumulation (data not shown) which was consistent with a previous publication [83]. Although the histological injury score of livers and kidneys along with survival rate in our LPS injection model did not improve by BAM15 possibly due to the nonlethality of the model, our result is a proof of concept supporting BAM15 as an interesting candidate for the sepsis anti-inflammatory drug. More studies are warranted.

In conclusion, an anti-inflammatory property of a mitochondrial uncoupling agent (BAM15) was demonstrated as an example of anti-inflammatory induction through an interference of the cell metabolic profile. BAM15 at- tenuated the severity of inflammation through the reduction of energy status in macrophages and hepatocytes. Further studies on the control of immune responses through cell metabolism in sepsis should be interesting.

\section{Acknowledgement}

We would like to thank Peerapat Visitchanakul for kindly helping in the research document.

\section{Statement of Ethics}

The animal procedure, in accordance with the protocol of the National Institutes of Health (NIH; USA), was approved by the Institutional Animal Care and Use Committee of the Faculty of Medicine, Chulalongkorn University, Bangkok, Thailand.

\section{Conflict of Interest Statement}

The authors have no conflicts of interest to disclose.

\section{Funding Sources}

This work was supported by the Rachadapiseksomphoch Endowment Fund and the Program Management Unit for Human Resources and Institutional Development Research and Innovation-CU (Global Partnership B16F630071 and Flagship B05F630073), TSRI Fund (CU_FRB640001_01_23_1), and the 90th Anniversary of Chulalongkorn University Fund (Ratchadapisek Sompote Endowment Fund). Cong Phi Dang was supported by the scholarship from the 100th Anniversary Chulalongkorn University Fund for Doctoral Scholarship.

\section{Author Contributions}

Conceptualization: C.P.D. and A.L.; methodology: C.P.D., K.U., T.B., W.S., and K.S.-K.; investigation: C.P.D., J.I.-A., A.C., and A.L.; writing, original draft preparation: C.P.D. and A.L.; writing, review and editing: C.P.D. and A.L.; supervision: A.L.; and funding acquisition: A.L.

\section{References}

1 Singer M, Deutschman CS, Seymour CW, Shankar-Hari M, Annane D, Bauer M, et al. The third international consensus definitions for sepsis and septic shock (sepsis-3). JAMA. 2016;315(8):801-10.

2 Doi K, Leelahavanichkul A, Yuen PS, Star RA. Animal models of sepsis and sepsis-induced kidney injury. J Clin Invest. 2009;119(10): 2868-78.
3 Rosen DA, Seki SM, Fernandez-Castaneda A, Beiter RM, Eccles JD, Woodfolk JA, et al. Modulation of the sigma-1 receptor-IRE1 pathway is beneficial in preclinical models of inflammation and sepsis. Sci Transl Med. 2019;11(478):eaau5266.

4 Riedemann NC, Ward PA. Anti-inflammatory strategies for the treatment of sepsis. Expert Opin Biol Ther. 2003;3(2):339-50.
5 Hotchkiss RS, Moldawer LL, Opal SM, Reinhart K, Turnbull IR, Vincent JL. Sepsis and septic shock. Nat Rev Dis Primers. 2016;2: 16045.

6 Mosser DM, Hamidzadeh K, Goncalves R Macrophages and the maintenance of homeostasis. Cell Mol Immunol. 2020.

7 Murray PJ. Macrophage polarization. Annu Rev Physiol. 2017;79:541-66.
BAM15 Attenuates Inflammation in Macrophages and Hepatocytes
J Innate Immun 2021;13:359-375 DOI: $10.1159 / 000516348$ 
8 Dang CP, Leelahavanichkul A. Over-expression of miR-223 induces M2 macrophage through glycolysis alteration and attenuates LPS-induced sepsis mouse model, the cellbased therapy in sepsis. PLoS One. 2020; 15(7):e0236038.

9 Taratummarat S, Sangphech N, Vu CTB, Palaga $\mathrm{T}$, Ondee $\mathrm{T}$, Surawut $\mathrm{S}$, et al. Gold nanoparticles attenuates bacterial sepsis in cecal ligation and puncture mouse model through the induction of M2 macrophage polarization. BMC Microbiol. 2018;18(1):85.

10 O'Neill LA, Kishton RJ, Rathmell J. A guide to immunometabolism for immunologists. Nat Rev Immunol. 2016;16(9):553-65.

11 Domblides C, Lartigue L, Faustin B. Metabolic stress in the immune function of $\mathrm{T}$ cells, macrophages and dendritic cells. Cells. 2018; 7(7):68.

12 Thapa B, Lee K. Metabolic influence on macrophage polarization and pathogenesis. $\mathrm{BMB}$ Rep. 2019;52(6):360-72.

13 Palsson-McDermott EM, O'Neill LAJ. Targeting immunometabolism as an anti-inflammatory strategy. Cell Res. 2020;30(4):300-14.

14 Gong Y, Lan H, Yu Z, Wang M, Wang S, Chen $\mathrm{Y}$, et al. Blockage of glycolysis by targeting PFKFB3 alleviates sepsis-related acute lung injury via suppressing inflammation and apoptosis of alveolar epithelial cells. Biochem Biophys Res Commun. 2017;491(2):522-9.

15 Ramond E, Jamet A, Coureuil M, Charbit A. Pivotal role of mitochondria in macrophage response to bacterial pathogens. Front Immunol. 2019;10:2461.

16 Dreschers S, Ohl K, Lehrke M, Mollmann J, Denecke B, Costa I, et al. Impaired cellular energy metabolism in cord blood macrophages contributes to abortive response toward inflammatory threats. Nat Commun. 2019; 10(1): 1685 .

17 Lee JH, Zhang Y, Zhao Z, Ye X, Zhang X, Wang $H$, et al. Intracellular ATP in balance of pro- and anti-inflammatory cytokines in adipose tissue with and without tissue expansion. Int J Obes. 2017;41(4):645-51.

18 Demine S, Renard P, Arnould T. Mitochondrial uncoupling: a key controller of biological processes in physiology and diseases. Cells. 2019;8(8):795.

19 Cadenas S. Mitochondrial uncoupling, ROS generation and cardioprotection. Biochim Biophys Acta Bioenerg. 2018;1859(9):940-50.

20 Brennan JP, Berry RG, Baghai M, Duchen MR, Shattock MJ. FCCP is cardioprotective at concentrations that cause mitochondrial oxidation without detectable depolarisation. Cardiovasc Res. 2006;72(2):322-30.

21 Grasmick KA, Hu H, Hone EA, Farooqi I, Rellick SL, Simpkins JW, et al. Uncoupling of the electron transport chain compromises mitochondrial oxidative phosphorylation and exacerbates stroke outcomes. J Neuroinfect Dis. 2018;9(4):283.

22 Sugiyama Y, Shudo T, Hosokawa S, Watanabe A, Nakano M, Kakizuka A. Emodin, as a mitochondrial uncoupler, induces strong de- creases in adenosine triphosphate (ATP) levels and proliferation of B16F10 cells, owing to their poor glycolytic reserve. Genes Cells. 2019;24(8):569-84.

23 Luo Y, Bond JD, Ingram VM. Compromised mitochondrial function leads to increased cytosolic calcium and to activation of MAP kinases. Proc Natl Acad Sci U S A. 1997;94(18): 9705-10.

24 Nicholls DG. The physiological regulation of uncoupling proteins. Biochim Biophys Acta. 2006 May-Jun;1757(5-6):459-66.

25 Kizaki T, Suzuki K, Hitomi Y, Taniguchi N, Saitoh D, Watanabe K, et al. Uncoupling protein 2 plays an important role in nitric oxide production of lipopolysaccharide-stimulated macrophages. Proc Natl Acad Sci U S A. 2002; 99(14):9392-7.

26 Haines B, Li PA. Overexpression of mitochondrial uncoupling protein 2 inhibits inflammatory cytokines and activates cell survival factors after cerebral ischemia. PLoS One. 2012;7(2):e31739.

27 Bai Y, Onuma H, Bai X, Medvedev AV, Misukonis $\mathrm{M}$, Weinberg JB, et al. Persistent nuclear factor-kappa B activation in Ucp2/ mice leads to enhanced nitric oxide and inflammatory cytokine production. J Biol Chem. 2005; 280(19):19062-9.

28 Kenwood BM, Weaver JL, Bajwa A, Poon IK, Byrne FL, Murrow BA, et al. Identification of a novel mitochondrial uncoupler that does not depolarize the plasma membrane. Mol Metab. 2014;3(2):114-23.

29 Axelrod CL, King WT, Davuluri G, Noland RC, Hall J, Hull M, et al. BAM15-mediated mitochondrial uncoupling protects against obesity and improves glycemic control. Embo Mol Med. 2020;12(7):e12088.

30 Alexopoulos SJ, Chen SY, Brandon AE, Salamoun JM, Byrne FL, Garcia CJ, et al. Mitochondrial uncoupler BAM15 reverses dietinduced obesity and insulin resistance in mice. Nat Commun. 2020;11(1):2397.

31 Yan J, Li S, Li S. The role of the liver in sepsis. Int Rev Immunol. 2014;33(6):498-510.

32 Leelahavanichkul A, Worasilchai N, Wannalerdsakun S, Jutivorakool K, Somparn P, Issara-Amphorn J, et al. Gastrointestinal leakage detected by serum $(1>3)$-beta-D-glucan in mouse models and a pilot study in patients with sepsis. Shock. 2016;46(5):506-18.

33 Ondee T, Gillen J, Visitchanakun P, Somparn $\mathrm{P}$, Issara-Amphorn J, Dang Phi C, et al. Lipocalin-2 (Lcn-2) attenuates polymicrobial sepsis with LPS preconditioning (LPS tolerance) in FcGRIIb deficient lupus mice. Cells. 2019; 8(9):1064.

34 Panpetch W, Hiengrach P, Nilgate S, Tumwasorn S, Somboonna N, Wilantho A, et al. Additional Candida albicans administration enhances the severity of dextran sulfate solution induced colitis mouse model through leaky gut-enhanced systemic inflammation and gut-dysbiosis but attenuated by Lactobacillus rhamnosus L34. Gut Microbes. 2020;11(3): 465-80.
35 Jaroonwitchawan T, Visitchanakun P, Dang PC, Ritprajak P, Palaga T, Leelahavanichkul A. Dysregulation of lipid metabolism in macrophages is responsible for severe endotoxin tolerance in FcgRIIB-deficient lupus mice. Front Immunol. 2020;11:959.

$36 \mathrm{Vu}$ CTB, Thammahong A, Leelahavanichkul A, Ritprajak P. Alteration of macrophage immune phenotype in a murine sepsis model is associated with susceptibility to secondary fungal infection. Asian Pac J Allergy Immunol. 2019.

$37 \mathrm{Vu}$ CTB, Thammahong A, Yagita H, Azuma M, Hirankarn N, Ritprajak P, et al. Blockade Of PD-1 attenuated postsepsis aspergillosis via the activation of IFN- $\gamma$ and the dampening of IL-10. Shock. 2020;53(4):514-24.

38 Song P, Zhang J, Zhang Y, Shu Z, Xu P, He L, et al. Hepatic recruitment of CD11b+Ly6C+ inflammatory monocytes promotes hepatic ischemia/reperfusion injury. Int J Mol Med. 2018;41(2):935-45

39 Mikula M, Majewska A, Ledwon JK, Dzwonek A, Ostrowski J. Obesity increases histone $\mathrm{H} 3$ lysine 9 and 18 acetylation at Tnfa and $\mathrm{Ccl} 2$ genes in mouse liver. Int J Mol Med. 2014;34(6):1647-54.

40 Zhang N, Weber A, Li B, Lyons R, Contag PR, Purchio AF, et al. An inducible nitric oxide synthase-luciferase reporter system for in vivo testing of anti-inflammatory compounds in transgenic mice. J Immunol. 2003;170(12):6307-19.

41 Panpetch W, Somboonna N, Palasuk M, Hiengrach P, Finkelman M, Tumwasorn S, et al. Oral candida administration in a clostridium difficile mouse model worsens disease severity but is attenuated by bifidobacterium. PLoS One. 2019;14(1):e0210798.

42 Issara-Amphorn J, Chancharoenthana W, Visitchanakun P, Leelahavanichkul A. Syk inhibitor attenuates polymicrobial sepsis in FcgRIIb-deficient lupus mouse model, the impact of lupus characteristics in sepsis. J Innate Immun. 2020;12(6):461-79.

43 Panpetch W, Sawaswong V, Chanchaem P, Ondee T, Dang CP, Payungporn S, et al. Candida administration worsens cecal ligation and puncture-induced sepsis in obese mice through gut dysbiosis enhanced systemic inflammation, impact of pathogen-associated molecules from gut translocation and saturated fatty acid. Front Immunol. 2020;11: 561652.

44 Gray LR, Tompkins SC, Taylor EB. Regulation of pyruvate metabolism and human disease. Cell Mol Life Sci. 2014;71(14):2577-604.

45 Amornphimoltham P, Yuen PST, Star RA, Leelahavanichkul A. Gut leakage of fungalderived inflammatory mediators: part of a gut-liver-kidney axis in bacterial sepsis. Dig Dis Sci. 2019;64(9):2416-28.

46 Leelahavanichkul A, Somparn P, Panich T, Chancharoenthana W, Wongphom J, Pisitkun T, et al. Serum miRNA-122 in acute liver injury induced by kidney injury and sepsis in CD-1 mouse models. Hepatol Res. 2015; 45(13):1341-52. 
47 Soto-Heredero G, Gomez de Las Heras MM, Gabande-Rodriguez E, Oller J, Mittelbrunn M. Glycolysis: a key player in the inflammatory response. FEBS J. 2020;287(16):3350-69.

48 Rogatzki MJ, Ferguson BS, Goodwin ML, Gladden LB. Lactate is always the end product of glycolysis. Front Neurosci. 2015;9:22.

49 Walesky CM, Kolb KE, Winston CL, Henderson J, Kruft B, Fleming I, et al. Functional compensation precedes recovery of tissue mass following acute liver injury. Nat Commun. 2020;11(1):5785

50 Nishikawa T, Bellance N, Damm A, Bing H, Zhu Z, Handa K, et al. A switch in the source of ATP production and a loss in capacity to perform glycolysis are hallmarks of hepatocyte failure in advance liver disease. J Hepatol. 2014;60(6):1203-11.

51 Sitia $G$, Iannacone $M$, Aiolfi R, Isogawa $M$, van Rooijen N, Scozzesi C, et al. Kupffer cells hasten resolution of liver immunopathology in mouse models of viral hepatitis. PLoS Pathog. 2011;7(6):e1002061.

52 Vollmar B, Menger MD. The hepatic microcirculation: mechanistic contributions and therapeutic targets in liver injury and repair. Physiol Rev. 2009;89(4):1269-339.

53 Garcia D, Shaw RJ. AMPK: mechanisms of cellular energy sensing and restoration of metabolic balance. Mol Cell. 2017;66(6):789800.

54 Carling D, Thornton C, Woods A, Sanders MJ. AMP-activated protein kinase: new regulation, new roles? Biochem J. 2012;445(1):1127.

55 Busiello RA, Savarese S, Lombardi A. Mitochondrial uncoupling proteins and energy metabolism. Front Physiol. 2015;6:36.

56 Holmuhamedov EL, Jovanovic S, Dzeja PP, Jovanovic A, Terzic A. Mitochondrial ATPsensitive $\mathrm{K}+$ channels modulate cardiac mitochondrial function. Am J Physiol. 1998; 275(5):H1567-76.

57 Emre Y, Hurtaud C, Karaca M, Nubel T, Zavala F, Ricquier $\mathrm{D}$. Role of uncoupling protein UCP2 in cell-mediated immunity: how macrophage-mediated insulitis is accelerated in a model of autoimmune diabetes. Proc Natl Acad Sci U S A. 2007;104(48):19085-90.

58 Feng J, Li J, Wu L, Yu Q, Ji J, Wu J, et al. Emerging roles and the regulation of aerobic glycolysis in hepatocellular carcinoma. J Exp Clin Cancer Res. 2020;39(1):126.

59 Nemeth K, Leelahavanichkul A, Yuen PS, Mayer B, Parmelee A, Doi K, et al. Bone marrow stromal cells attenuate sepsis via prostaglandin $\mathrm{E}(2)$-dependent reprogramming of host macrophages to increase their interleukin-10 production. Nat Med. 2009;15(1):429.
60 Tang M, Luo Z, Wu Y, Zhuang J, Li K, Hu D, et al. BAM15 attenuates transportation-induced apoptosis in iPS-differentiated retinal tissue. Stem Cell Res Ther. 2019;10(1):64

61 Ishisaka A, Kawabata K, Miki S, Shiba Y, Minekawa S, Nishikawa T, et al. Mitochondrial dysfunction leads to deconjugation of quercetin glucuronides in inflammatory macrophages. PLoS One. 2013;8(11):e80843.

62 Nomura J, So A, Tamura M, Busso N. Intracellular ATP decrease mediates NLRP3 inflammasome activation upon nigericin and crystal stimulation. J Immunol. 2015;195(12): 5718-24.

63 McCall CE, Zabalawi M, Liu T, Martin A, Long DL, Buechler NL, et al. Pyruvate dehydrogenase complex stimulation promotes immunometabolic homeostasis and sepsis survival. JCI Insight. 2018;3(15):e99292.

64 Stacpoole PW, Wright EC, Baumgartner TG, Bersin RM, Buchalter S, Curry SH, et al. A controlled clinical trial of dichloroacetate for treatment of lactic acidosis in adults. The dichloroacetate-lactic acidosis study group. $\mathrm{N}$ Engl J Med. 1992;327(22):1564-9.

65 Grondman I, Arts RJW, Koch RM, Leijte GP, Gerretsen J, Bruse N, et al. Frontline science: endotoxin-induced immunotolerance is associated with loss of monocyte metabolic plasticity and reduction of oxidative burst. J Leukoc Biol. 2019;106(1):11-25.

66 Ondee T, Surawut S, Taratummarat S, Hirankarn N, Palaga T, Pisitkun P, et al. Fc gamma receptor IIB deficient mice: a lupus model with increased endotoxin tolerance-related sepsis susceptibility. Shock. 2017;47(6):74352.

67 Alasadi A, Chen M, Swapna GVT, Tao H, Guo J, Collantes J, et al. Effect of mitochondrial uncouplers niclosamide ethanolamine (NEN) and oxyclozanide on hepatic metastasis of colon cancer. Cell Death Dis. 2018;9(2): 215.

68 De Simone R, Ajmone-Cat MA, Pandolfi M, Bernardo A, De Nuccio C, Minghetti L, et al. The mitochondrial uncoupling protein-2 is a master regulator of both M1 and M2 microglial responses. J Neurochem. 2015;135(1): $147-56$.

69 Basu Ball W, Kar S, Mukherjee M, Chande AG, Mukhopadhyaya R, Das PK. Uncoupling protein 2 negatively regulates mitochondrial reactive oxygen species generation and induces phosphatase-mediated anti-inflammatory response in experimental visceral leishmaniasis. J Immunol. 2011;187(3):1322-32.

70 Doi K. Role of kidney injury in sepsis. J Intensive Care. 2016;4:17

71 Poston JT, Koyner JL. Sepsis associated acute kidney injury. BMJ. 2019;364:k4891.
72 Schieber AMP, Ayres JS. Thermoregulation as a disease tolerance defense strategy. Pathog Dis. 2016;74(9):ftw106.

73 Sag D, Carling D, Stout RD, Suttles J. Adenosine 5'-monophosphate-activated protein kinase promotes macrophage polarization to an anti-inflammatory functional phenotype. J Immunol. 2008;181(12):8633-41.

74 Tai Y, Li L, Peng X, Zhu J, Mao X, Qin N, et al. Mitochondrial uncoupler BAM15 inhibits artery constriction and potently activates AMPK in vascular smooth muscle cells. Acta Pharm Sin B. 2018;8(6):909-18.

75 Escobar DA, Botero-Quintero AM, Kautza BC, Luciano J, Loughran P, Darwiche S, et al. Adenosine monophosphate-activated protein kinase activation protects against sepsisinduced organ injury and inflammation. J Surg Res. 2015;194(1):262-72.

76 Zhang M, Yang D, Gong X, Ge P, Dai J, Lin L, et al. Protective benefits of AMP-activated protein kinase in hepatic ischemia-reperfusion injury. Am J Transl Res. 2017;9(3):8239.

77 Zhao W, Zhang L, Chen R, Lu H, Sui M, Zhu $\mathrm{Y}$, et al. SIRT3 protects against acute kidney injury via AMPK/mTOR-regulated autophagy. Front Physiol. 2018;9:1526.

78 Tong X, Smith KA, Pelling JC. Apigenin, a chemopreventive bioflavonoid, induces AMP-activated protein kinase activation in human keratinocytes. Mol Carcinog. 2012; 51(3):268-79.

79 Zhao X, Zmijewski JW, Lorne E, Liu G, Park YJ, Tsuruta Y, et al. Activation of AMPK attenuates neutrophil proinflammatory activity and decreases the severity of acute lung injury. Am J Physiol Lung Cell Mol Physiol. 2008; 295(3):L497-504.

80 Yun H, Park S, Kim MJ, Yang WK, Im DU, Yang KR, et al. AMP-activated protein kinase mediates the antioxidant effects of resveratrol through regulation of the transcription factor FoxO1. FEBS J. 2014;281(19):4421-38.

81 Alba G, El Bekay R, Alvarez-Maqueda M, Chacon P, Vega A, Monteseirin J, et al. Stimulators of AMP-activated protein kinase inhibit the respiratory burst in human neutrophils. FEBS Lett. 2004;573(1-3):219-25.

82 Ido Y, Carling D, Ruderman N. Hyperglycemia-induced apoptosis in human umbilical vein endothelial cells: inhibition by the AMPactivated protein kinase activation. Diabetes. 2002;51(1):159-67.

83 Chung KW, Kim KM, Choi YJ, An HJ, Lee B, $\mathrm{Kim} \mathrm{DH}$, et al. The critical role played by endotoxin-induced liver autophagy in the maintenance of lipid metabolism during sepsis. Autophagy. 2017;13(7):1113-29.
BAM15 Attenuates Inflammation in Macrophages and Hepatocytes
J Innate Immun 2021:13:359-375 